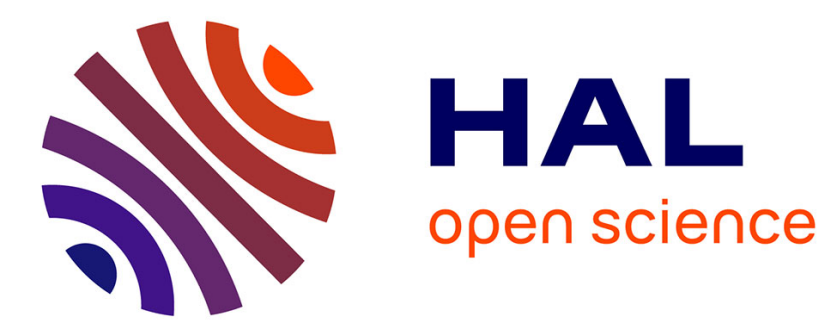

\title{
Very low energy photoelectron spectroscopy in multiphoton ionization of $\mathrm{H} 2$
}

\author{
C. Bordas, M. Dyer, H. Helm
}

\section{To cite this version:}

C. Bordas, M. Dyer, H. Helm. Very low energy photoelectron spectroscopy in multiphoton ionization of H2. Journal de Physique IV Proceedings, 1994, 04 (C4), pp.C4-691-C4-694. 10.1051/jp4:19944182 . jpa-00252642

\section{HAL Id: jpa-00252642 https://hal.science/jpa-00252642}

Submitted on 1 Jan 1994

HAL is a multi-disciplinary open access archive for the deposit and dissemination of scientific research documents, whether they are published or not. The documents may come from teaching and research institutions in France or abroad, or from public or private research centers.
L'archive ouverte pluridisciplinaire HAL, est destinée au dépôt et à la diffusion de documents scientifiques de niveau recherche, publiés ou non, émanant des établissements d'enseignement et de recherche français ou étrangers, des laboratoires publics ou privés. 


\title{
Very low energy photoelectron spectroscopy in multiphoton ionization of $\mathrm{H}_{2}$
}

\author{
C. BORDAS*, M.J. DYER and H. HELM
}

\author{
Molecular Physics Laboratory, SRI International, 333 Ravenswood Avenue, Menlo Park, CA 94025, \\ U.S.A. \\ * Laboratoire de Spectrométrie Ionique et Moléculaire, URA 171 du CNRS, Université Lyon I, Bât. 205, \\ 43 Bd du 11 Novembre 1918, 69622 Villeurbanne cedex, France
}

\begin{abstract}
We apply a novel photoelectron imaging spectrometer [1] to the energy and angular analysis of photoelectrons resulting from resonantly enhanced multiphoton ionization of $\mathrm{H}_{2}$. Photoelectron images with well resolved individual rotational structure of the resulting $\mathrm{H}_{2}{ }^{+}$ion are obtained, demonstrating the capability of this technique for analyzing photoelectrons in the meV energy range.
\end{abstract}

\section{I - Introduction.}

Resonantly enhanced multiphoton ionization (REMPI) of molecules has been widely used to study neutral excited states. Coupled with mass analysis of fragment ions, this method allows the investigation of the fragmentation mechanisms of the excited neutral and ionic species. A finer analysis may be achieved if the kinetic energy of the ejected electrons is measured, allowing the determination of the branching ratios among the different rovibrational or electronically excited ionization channels. Photoelectron spectroscopy has been applied successfully to a large number of molecules. Molecular hydrogen, owing to its intrinsic simplicity, and to the detailed knowledge of its excited states, has been the most widely studied [2-5].

Conventional photoelectron spectrometry using time-of-flight or magnetic bottles presents limitations, especially for low energy electrons. Here we describe the operation of a spectrometer that combines efficient detection with good energy resolution and the ability to monitor angular distributions for photoelectrons in the meV range.

\section{II - Experimental set-up.}

The basic principle of the imaging spectrometer (figure 1) is to project the electronic cloud resulting from the ionization process onto a two-dimensional position sensitive detector. The resulting image contains information on the initial kinetic energy and angular distribution. Photoelectrons generated at a point source in a field-free region with a discrete energy $W$ at time $t=0$, are later found on the surface of a sphere of radius $R(t)=t .(2 W / m)^{1 / 2}$. For instance, electrons ejected with an initial energy of $1 \mathrm{eV}$, are found, after $20 \mathrm{ns,} \mathrm{on} \mathrm{the} \mathrm{surface} \mathrm{of} \mathrm{a} \mathrm{sphere} \mathrm{of}$ $12 \mathrm{~mm}$ radius. In our experiment a homogeneous, static electric field is applied in the ionization region. As a result, the expanding photoelectron cloud is projected onto a position sensitive detector situated perpendicular to the electric field. The detector is a pair of multichannel plates of $5 \mathrm{~cm}$ diameter, coupled with a phosphor screen. This results in a circular optical image of electron impacts with a maximum intensity at radius: $R=2(\mathrm{LW} / \mathrm{qF})^{1 / 2}$, where $\mathrm{L}$ is the distance between the ionization point source and $\mathrm{F}$ is the electric field strength. The filling pattern of the circular image reflects the spatial distribution of the photoelectrons immediately after ionization. The screen phosphorescence is recorded via a CCD camera and digitized in a microcomputer. A statistically meaningful image is obtained by digitally summing several thousand laser shots, each shot capturing typically 10 to 100 electrons. This number is deliberately kept at a low value in order to avoid saturation of the detector and space charge effects. 
Control of the number of photoelectrons produced is via the gas pressure and laser intensity. The base pressure in the chamber is kept below $10^{-9}$ Torr in order to avoid ionization of residual compounds. A REMPI process induced by a single linearly polarized light source leads to an electron emission pattern that is rotationally symmetric around the laser polarization. Hence, the planar image recorded on a position sensitive detector parallel to the laser polarization can be deconvoluted by an Abel inversion, giving directly the initial velocity and angular distribution of the photoelectrons.

The resolution of the detector is governed by several parameters that can be adjusted to the experimental conditions: (i) the size of the ionization volume determines the maximum achievable resolu-

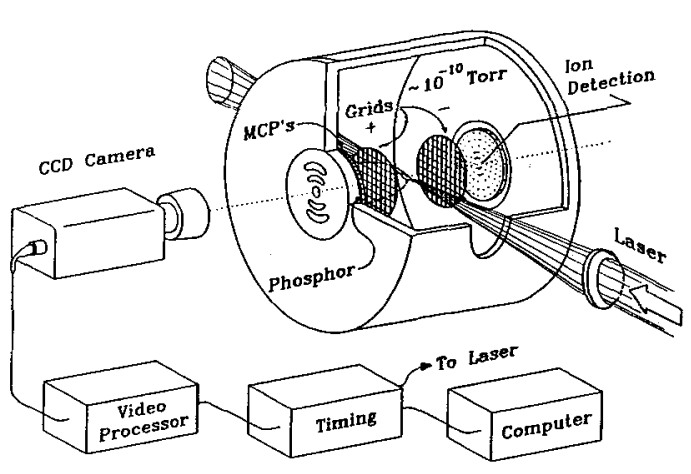

Figure 1: Schematic of the photoelectron imaging spectrometer tion; (ii) the spatial resolution of the detector, primarily determined by the camera pixel geometry. The resolution in electron velocity is linearly related to the resolution of the image radius. Hence, it is desirable to adjust the projection field, F, such that an image of largest possible diameter appears on the detector.

\section{III - Results.}

We use the imaging spectrometer to study the three-photon resonance-enhanced, four-photon ionization of $\mathrm{H}_{2}$ with selected intermediate levels of the B ${ }^{1} \Sigma_{u}{ }^{+}$and $C^{1} \Pi_{u}$ states (figure 2), using a tunable UV laser of $5 \mathrm{~ns}$ duration, with focal intensities in the range $1-5 \mathrm{GW} / \mathrm{cm}^{2}$. These transitions have been studied by several authors in the past [2-5]. The present work is concerned especially with the formation of very low energy photoelectrons, and their associated angular distribution.

Figure 3 shows a typical experimental result obtained when exciting the $B{ }^{1} \Sigma_{u^{+}}\left(v^{\prime}=3, J^{\prime}=2\right)$ intermediate via the $3 \mathrm{~h} v \mathrm{R} 1$ transition. The raw image is presented in figure 3-a. After the Abel inversion, one can obtain the velocity spectrum (3-b) and the angular distribution in the different ionization channels(3-c). In that case, the spectrum is fully resolved and each channel may be analysed separately. Two competing processes appear:

- (1) molecular ionization:

$$
\mathrm{H}_{2}+3 \mathrm{hv} \rightarrow \mathrm{H}_{2}\left(\mathrm{~B}^{1} \Sigma_{\mathrm{u}^{+}}, \mathrm{v}^{\prime}=3, \mathrm{~J}^{\prime}=2\right) \text { followed by } \mathrm{H}_{2}{ }^{*}+\mathrm{hv} \rightarrow \mathrm{H}_{2}{ }^{+}\left(\mathrm{v}^{+}=0 ; \mathrm{N}^{+}=1,3 \text {, or } 5\right)+\mathrm{e}^{-}
$$

This process gives rise to three different ionization channels corresponding to photoelectrons of 126,91 , and 27 meV respectively. The three rotational final channels appear as the three inner ring structures in the raw image. 
- and (2) molecular dissociation followed by one-photon ionization of the excited atom:

$$
\begin{array}{ll} 
& \mathrm{H}_{2}+3 \mathrm{hv} \rightarrow \mathrm{H}_{2}\left(\mathrm{~B}^{1} \Sigma_{\mathrm{u}}{ }^{+}, \mathrm{v}^{\prime}=3, \mathrm{~J}^{\prime}=2\right) \\
\text { followed by photodissociation } & \left.\mathrm{H}_{2}\left(\mathrm{~B}^{1} \Sigma_{\mathrm{u}}{ }^{+}, \mathrm{v}^{\prime}=3, \mathrm{~J}^{\prime}=2\right)+\mathrm{h} v \rightarrow \mathrm{H}(1 \mathrm{~s})+\mathrm{H}\right) \\
\text { and then a fifth photon ionizes the atom } & \mathrm{H}(\mathrm{n}=2, \ell)+\mathrm{h} v \rightarrow \mathrm{H}^{+}+\mathrm{e}^{-}
\end{array}
$$

The fourth-photon step is in direct competition with the molecular ionization channel. The photoelectrons emitted in this process have a kinetic energy of $487 \mathrm{meV}$. Contributions in the $\mathrm{H}(\mathrm{n}=3)$ channel would require two-photon absorption from the B state and are not observed for this transition.

Figure 3: Experimental results obtained with the $B^{1} \Sigma_{u}{ }^{+}\left(v^{\prime}=3, J^{\prime}=2\right)$ intermediate level (RI line). (a) raw image; (b) velocity spectrum; (c) angular distributions: squares : $H(n=2)$ channel; circles: $v^{+}=0, N^{+}=1$ channel; triangles: $v^{+}=0, N^{+}=5$ channel.

(a)

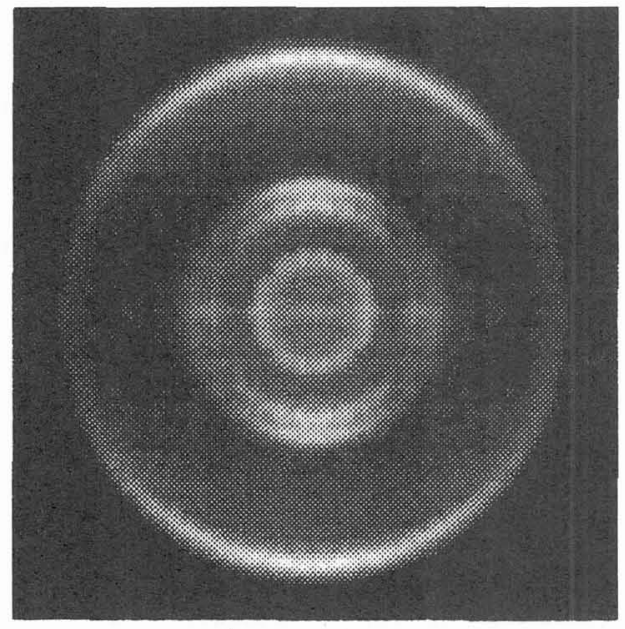

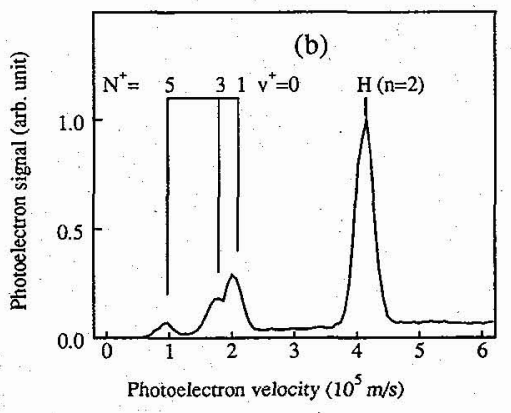

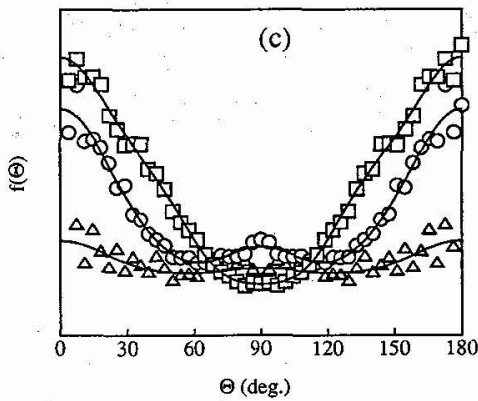

The angular distributions in the various ionization channels are displayed in figure 3-c. The full lines are the result of a fit using the expression:

$$
f(\Theta)=\sum_{i=0}^{4} \beta_{2 i} \cdot \cos ^{2 i}(\Theta)
$$

relevant to a four-photon ionization process.

In the case of the $B\left(v^{\prime}=3, J^{\prime}=2\right)$ intermediate which is ionized by one photon, only the $v^{+}=0$ channel is open. The magnitude of the dissociation signal is related to the overlap of the B state wavefunction with the continuum of the $\mathrm{Q}_{1}$ state. In the present case, the dissociation channel is largely dominant. Similar results are displayed in figure 4 , using the $B{ }^{1} \Sigma_{u}{ }^{+}\left(v^{\prime}=4, J^{\prime}=0\right)$ intermediate level. In that case, five ionization channels are open at $2 \mathrm{meV}$ and $39 \mathrm{meV}$ for $\mathrm{v}^{+}=1$ (resp. $\mathrm{N}^{+}=3$ and 1 ), 274 and $310 \mathrm{meV}$ for $\mathrm{v}^{+}=0$ (resp. $\mathrm{N}^{+}=3$ and 1 ), and $533 \mathrm{meV}$ for $\mathrm{H}(\mathrm{n}=2)$. Note that the slow electron peak at $2 \mathrm{meV}$ is fully resolved while the two rotational branches are not distinguishable in the $\mathrm{v}^{+}=0$ peak. This is a result of the fact that the absolute velocity resolution of the spectrometer is a constant fraction of the initial electron velocity. As compared to previous results [4], there is an improvement in resolution at low energy, while the present resolution is lower above $0.5 \mathrm{eV}$. Although this resolution could be improved by using a larger detector and a higher resolution camera the main advantage is that rotationally resolved angular distributions can be obtained in the very low energy range. From this point of view, 
these preliminary results are very encouraging. The detailed analysis of these and similar observations is now under progress and will be published elsewhere.

Figure 4: Experimental results obtained with the $B^{1} \Sigma_{u}{ }^{+}\left(v^{\prime}=4, J^{\prime}=0\right)$ intermediate level ( $P 1$ line) (a) Abel-inverted image; (b) velocity spectrum.

(a)
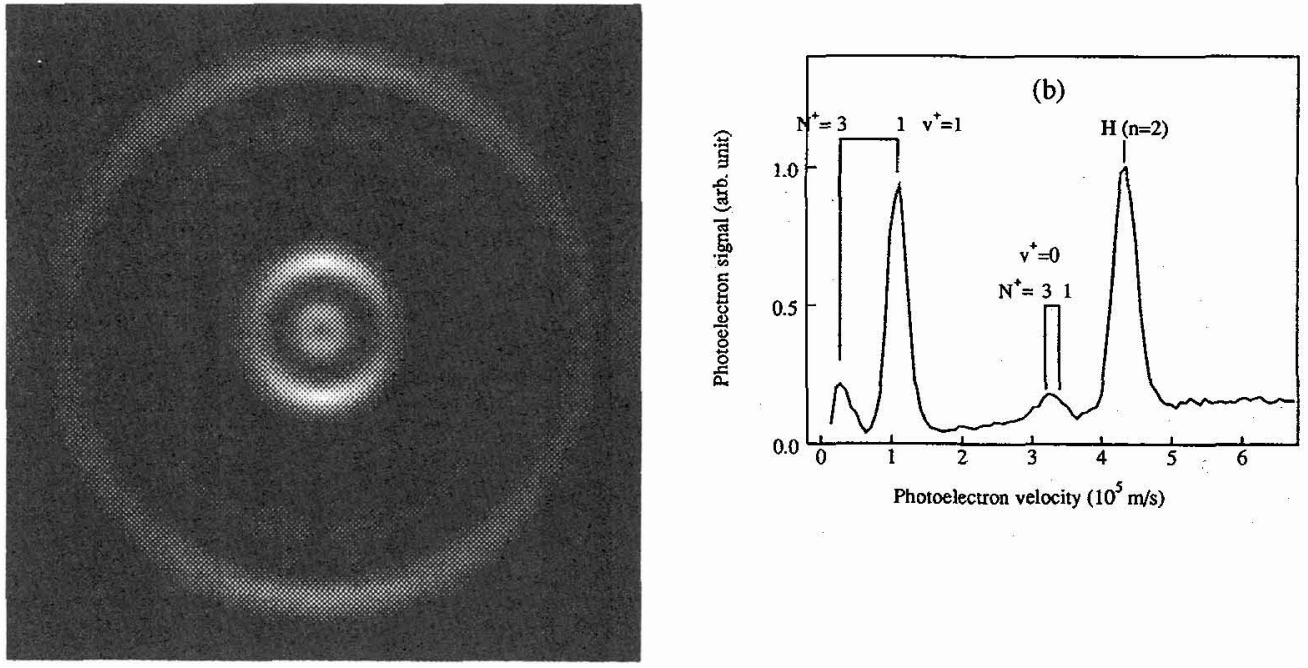

The same principle of imaging spectrometry may be applied to the ionic species. The geometry of the image does not depend on the mass of the charged particle, as opposed to time of flight techniques. Therefore, it is possible to record simultaneously photoelectrons and photoions with two $\mathrm{CCD}$ devices. The energetic and angular distribution can then be measured for all charged products. This opens a broad domain of applications to the imaging technique in the area of molecular or cluster physics.

This research was funded by the National Science Foundation under grant PHY-9249199. C. B. acknowledges continuous support from the "Centre National de la Recherche Scientifique".

\section{References:}

[1] H. Helm, N. Bjerre, M.J. Dyer, D.L. Huestis, and M. Saeed, Phys. Rev. Lett. 70, 3221 (1993).

[2] S.T. Pratt, P.M. Dehmer, and J.L. Dehmer, J. Chem. Phys. 78, 4315 (1983); Chem. Phys. Letters 105, 28 (1984).

[3] J.H.M. Bonnie, J.W. Verschuur, H.J. Hopman, and H.B. Van Linden Van Den Heuvell, Chem. Phys. Letters 130, 43 (1986).

[4] M.A. O'Halloran, S.T. Pratt, P.M. Dehmer, and J.L. Dehmer, J. Chem. Phys. 87, 3289 (1987).

[5] E.Y. Xu, T. Tsuboi, R. Kachru, and H. Helm, Phys. Rev. A 36, 5645 (1987). 